\title{
Correction to: Laccases in the Context of Potentially Cooperating Enzymes
}

\author{
Kamahldin Haghbeen and Dietmar Schlosser
}

\section{Correction to:}

Chapter 4 in: D. Schlosser (ed.), Laccases in Bioremediation and Waste Valorisation, Microbiology Monographs 33, https://doi.org/10.1007/978-3-030-47906-0_4

The original version of Chapter 4 was inadvertently published with incorrect last name of the author. The name "Dietmar Schlossser" has now been corrected to "Dietmar Schlosser". 